The Journal of the Acoustical Society of America

HOME

BROWSE

MORE $\nabla$

Full

Published Online: April 2016

\title{
Individual differences in hearing-impaired data: Stats, troubles, and approaches
}

\section{Sarah Verhulst}

View Affiliations

The Journal of the Acoustical Society of America 139, 2101 (2016); doi: http://dx.do i.org/10.1121/1.4950240
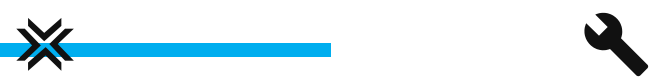

$\infty$

Il 


\section{ABSTRACT}

Individual differences in hearing ability might be dominated by subcomponents of hearing loss, e.g., cochlear gain loss, cochlear neuropathy, temporal coding deficits in low/high frequency regions, or combinations of these components. Unfortunately, we can only rely on indirect and hypothesis-driven objective (e.g., OAE/ABR/EFR) and psychoacoustic threshold metrics that aim to quantify these subcomponents of hearing loss, complicating a straightforward explanation of study results. Because correlations statistics often rely on small listener groups in which each data point could have resulted from different SNRs, metric-specific variability, it is not always clear which correlations are significant and meaningful. Additionally, multiple measures provide a multitude of correlations that should all support the common underlying hypothesis before conclusions can be drawn. In this tutorial, I provide some examples and approaches to more (and less) meaningful correlations based on recently collected objective and psychoacoustic measures in a group of normal and hearing-impaired listeners. Finally, I will introduce how computational model approaches might direct the interpretation of experimental results when several interacting sources of hearing impairment impact outcome measures unexpectedly. 


\section{Resources}

AUTHOR

LIBRARIAN

ADVERTISER

\section{General Information}

ABOUT

CONTACT

HELP

PRIVACY POLICY

TERMS OF USE

FOLLOW AIP PUBLISHING:

f $y$ in 